\title{
The rotational centroid and its application in quantum molecular dynamics simulations
}

\author{
L. HERNÁNDEZ DE LA PEÑA and P. G. KUSALIK* \\ Department of Chemistry, Dalhousie University, \\ Halifax, Nova Scotia, B3H 4J3, Canada \\ (Received 13 January 2004; revised version accepted 26 March 2004)
}

\begin{abstract}
The centroid molecular dynamics (CMD) method is extended to the rotational motion of rigid bodies. An algorithm is developed that homogeneously samples the orientational neighbourhood associated with the quantum degrees of freedom of a specified orientational centroid and by design ensures the constancy of the centroid. As part of this development a general definition for an orientational centroid (or average rotation), as well as a procedure to determine it, are presented. The application of this CMD methodology in quantum simulations of rigid body systems is discussed and demonstrated for a simple system.
\end{abstract}

\section{Introduction}

Feynman's path integral representation of statistical mechanics [1] has proven to be a very useful tool for studying quantum effects in condensed matter. This approach allows the use of Monte Carlo (MC) and molecular dynamics (MD) methods to study quantum mechanical effects in equilibrium and dynamical properties of complex systems, for example liquid water, excess electrons in liquids, etc. [2-4]. Within this context, centroid molecular dynamics (CMD) has been developed into a very successful technique for examining (approximate) quantum dynamics in condensed phases, becoming exact in the classical limit $[5,6]$. CMD has been justified formally and applied in the context of translational degrees of freedom (Cartesian coordinates) in atomic approaches [5-7]. However, in condensed phase phenomena, intramolecular vibrations are in many cases sufficiently decoupled from molecular translations and rotations that they have negligible impact on most bulk properties. The popularity of rigid models in water simulations, for example, relies on this very point, and their usefulness in the simulation of bulk aqueous systems has been extensively tested and confirmed [4, 8, 9].

As is well known from Feynman's work [1, 2], a quantum mechanical centroid density, $\rho_{\mathrm{c}}\left(x_{\mathrm{c}}\right)$, which is the path sum over all paths with their centroid located at the same point in space, $x_{c}$, can be defined for the path centroid variable, where the centroid variable is defined as the imaginary time average of a closed Feynman

*Author for correspondence. e-mail: peter.kusalik@dal.ca path. The centroid density formally defines a classicallike effective potential, $V_{\mathrm{c}}\left(x_{\mathrm{c}}\right)$, and the canonical partition function can then be obtained by the integration of the centroid density over all possible configurations of the centroid such that

$$
Z=\int \mathrm{d} x_{\mathrm{c}} \rho_{\mathrm{c}}\left(x_{\mathrm{c}}\right) \propto \int \mathrm{d} x_{\mathrm{c}} \exp \left[-\beta V_{\mathrm{c}}\left(x_{\mathrm{c}}\right)\right]
$$

The quantum dynamics can be (approximately) studied using the trajectories of the centroids that are generated by solving Newtonian-like equations of motions where the forces are derived from mean force potentials between centroids [5-7],

$$
m \ddot{x}(t)=-\frac{\mathrm{d} V_{\mathrm{c}}\left(x_{\mathrm{c}}\right)}{\mathrm{d} x_{\mathrm{c}}},
$$

in which $-\mathrm{d} V_{\mathrm{c}}\left(x_{\mathrm{c}}\right) / \mathrm{d} x_{\mathrm{c}}$ is a centroid constrained Feynman path average. The resulting centroid trajectory is then used to calculate equilibrium time correlation functions in a classical manner by averaging initial conditions over the centroid distribution function. If the position-position centroid correlation function, for example, is given by $\left\langle q_{\mathrm{c}}(0) q_{\mathrm{c}}(t)\right\rangle$, the usual quantum position correlation function, $C(t)=\langle q(0) q(t)\rangle$, can then be obtained by Fourier transform through the frequency space relationship [7],

$$
\tilde{C}(w)=\frac{\hbar \beta w}{2}\left[\operatorname{coth}\left(\frac{\hbar \beta w}{2}\right)+1\right] \tilde{C}^{*}(w),
$$


where $\tilde{C}^{*}(\omega)$ and $\tilde{C}(\omega)$ are the Fourier transforms of the centroid correlation function and the quantum time correlation function, respectively.

The advantage in extending the CMD methodology to the rotations of rigid bodies is that the number of degrees of freedom required in the simulation is decreased significantly:

(i) classically, by excluding the vibrational motion;

(ii) quantum mechanically, because treating the molecule as a rigid rotor allows for significantly lower values of the discretization parameter, $P$.

Consequently, the simulation is dramatically less expensive computationally without significant loss of physical insight. This approach also allows the dynamics of the translational degrees of freedom to be treated either classically or quantum mechanically according to the nature of the systems under consideration. The extension of the centroid methodology to rotations, while initially appearing straightforward, also has several subtleties that must be overcome, including the well-known noncommutativity property of finite rotations.

In this work we develop and discuss an approach that allows the implementation of the CMD method for treating explicitly the rotational uncertainty in the motion of a rigid body, applicable to condensed phase systems close to the classical limit (relatively high temperature). We present a centroid methodology applicable to the three-dimensional rotation of any arbitrary rigid object and demonstrate its successful application in the simulation of a simple molecular (water) system. Moreover, the requisite definition of an orientational centroid utilized in our approach may have numerous other applications that have explicit orientational dependence.

\section{Description of methodology}

\subsection{Translational $C M D$}

CMD resembles classical $\mathrm{MD}$ in many aspects and this similarity (which is particularly useful for present purposes) is better seen in the discretized picture. In the discretized picture the continuous path is approximated by $P$ beads, and this discretization parameter is chosen such that the calculation is sufficiently well converged to the $P \rightarrow \infty$ limiting result. The Hamiltonian for a discretized quantum particle can be written as [2]

$$
H=\sum_{t=1}^{P}\left(\frac{p_{t}^{2}}{2 m_{t}^{\prime}}+\frac{m P}{2 \hbar^{2} \beta^{2}}\left(x_{t}-x_{t+1}\right)^{2}+\frac{1}{P} V\left(x_{t}\right)\right),
$$

where $p_{t}$ and $x_{t}$ are the momenta and positions of the beads. The kinetic term is completely arbitrary (arbitrary moments and masses) and allows one to define the Hamiltonian in equation (4) with which to carry out the path integral evaluation via a molecular dynamics simulation. The second term in the Hamiltonian is the potential due to nearest neighbour harmonic coupling between beads which arises from the free particle part of the density matrix; this term is strictly of quantum origin. The last term is the interaction felt by each bead due to the external potential, where in condensed phase simulations this contribution is usually the intermolecular interaction. In writing equation (4) we have assumed we have only positional coordinates and translational motion.

In the discretized path integral picture (i.e. for finite $P)$, the path centroid variable is identical to the centre of mass of the isomorphic polymer of classical quasiparticles (or beads) such that $[6,7]$

$$
x_{0}=\frac{1}{P} \sum_{t=1}^{P} x_{t}
$$

Thus, a constraint is a necessary ingredient of the dynamics defined by the Hamiltonian in equation (4). A molecular dynamics algorithm designed to carry out this centroid-constrained path integral calculation can be described as follows. The total force acting on the bead $t, F_{t}$, can be expressed as $F_{t}=F^{\mathrm{q}}+F^{\mathrm{Int}}$, where $F^{\mathrm{q}}$ and $F^{\text {Int }}$ are the forces associated with the quantum and intermolecular potentials identified in equation (4), respectively. Furthermore, CMD defines the force acting on the centroid as

$$
F_{\mathrm{c}}=\frac{1}{P} \sum_{t=1}^{P} F_{t}
$$

It is then this force which must be averaged over the path fluctuations (i.e. in imaginary time). To obtain the force acting on the beads (this is the force that will govern the motion of the beads relative to the centroid) it is necessary to subtract the centroid force from the total force acting on the bead:

$$
F_{t}^{\text {bead }}=F_{t}-F_{\mathrm{c}}
$$

Thus, it becomes clear that this formulation of CMD is equivalent to requiring

$$
\sum_{t=1}^{P} F_{t}^{\text {bead }}=0
$$

Obviously this condition will conserve the total linear momentum of the $P$ beads, and if this sum is set to zero 
as an initial condition, i.e.

$$
\sum_{t=1}^{P} p_{t}=0
$$

the centroid or 'beads' centre of mass' will be a conserved quantity in the dynamics.

\subsection{Rotational CMD}

The treatment of the orientational case, even close to the classical limit, may at first appear far from obvious due to the lack of commutativity of the rotation operators (for example, equation (5) appears to have no clear analogue). However, we exploit a dynamical approach that takes advantage of the fact that infinitesimal rotation operators commute. Additionally, in our resolution of the problem, three frames are now required to describe rotations, the laboratory frame with respect to which the centroid moves, the centroid frame with respect to which the beads move and the local frames of the beads. (In the classical limit, it is clear that the centroid frame becomes the molecular principal frame and the analysis becomes identical to the standard two-frame rotational problem of a classical MD simulation.)

Our development begins by extending the translational centroid definition given above. The orientational centroid can be conserved dynamically by requiring the sum of the torques acting on the beads to be zero in the centroid frame,

$$
\sum_{t=1}^{P} T_{t}^{\text {bead }}=0
$$

and by imposing a constraint on the initial sum of the angular momenta in the centroid frame,

$$
\sum_{t=1}^{P} L_{t}=0
$$

It is instructive to analyse the consequences of these constraints for particular numbers of beads, where we assume as an initial condition that the beads are all aligned.

(i) $P=1$. As expected the classical limit is reached and the centroid describes the motion of the particles in a classical sense.

(ii) $P=2$. In this case, it is easily shown that $T_{1}=-T_{2}$ or that the torques acting on the beads exactly cancel. Equations ( $7 \mathrm{a}$ ) and (7 b) clearly allow the conservation of the centroid. (iii) $P>2$. In this case the interpretation of the constraint can no longer be easily pictured physically, due to the non-commutativity property of orientations. All that can be said is that the $P$ orientations will disperse with (imaginary) time while still satisfying the constraint.

We note that while the initial conditions allow us to specify a particular value for the centroid, the constraint of the orientational centroid has avoided a precise definition for (or an evolution of) the centroid.

The total torque acting on a bead, analogous to the translational case, has two contributions, one of a quantum nature and the other due to the external potential. It can be easily shown using the free orientational density matrix that at relatively high temperatures the potential between beads for the quantum rotational motion can be simplified to be $[4,10]$

$$
V \approx \frac{I P \Gamma_{i}^{2}(t+1, t)}{2 \hbar^{2} \beta^{2}},
$$

where $\Gamma(t, t+1)$ is the rotation angle (arc length) between the beads $t+1$ and $t$. We note that this simplification of the potential is not a requirement of the present algorithm. The arc length can be related to quaternion parameters by $\Gamma(t+1, t)=2 \arccos \left(\chi_{t+1}\right)$, where $\chi_{t+1}=q_{0}^{t+1} q_{0}^{t}+q_{1}^{t+1} q_{1}^{t}+q_{2}^{t+1} q_{2}^{t}+q_{3}^{t+1} q_{3}^{t}$ is the scalar element of the composite quaternion between $q^{t} \quad$ and $\quad q^{t+1}, \quad$ and $\quad q^{t} \equiv\left(q_{0}^{t}, q_{1}^{t}, q_{2}^{t}, q_{3}^{t}\right), \quad q^{t+1}=$ $\left(q_{0}^{t+1}, q_{1}^{t+1}, q_{2}^{t+1}, q_{3}^{t+1}\right)$ are the quaternion parameters that specify the orientations of the beads $t$ and $t+1$, respectively.

Using the fact that the principal torques, $T$, can be defined in quaternion form by [11]

$$
T=\frac{1}{2} \Xi \cdot N
$$

where explicit forms for $\Xi$ and $N$ are given in Appendix A. The torque acting on a particular bead $t$ can now be expressed as

$$
\begin{aligned}
T_{t}= & T^{(t+1)}+T^{(t-1)} \\
= & \frac{2 I P}{\hbar^{2} \beta^{2}} \Xi_{t}\left(\frac{\Gamma(t+1, t)}{\left(1-\chi_{t+1}^{2}\right)^{1 / 2}}\left(\frac{\partial \chi_{t+1}}{\partial q^{t+1}}\right)\right. \\
& \left.+\frac{\Gamma(t-1, t)}{\left(1-\chi_{t-1}^{2}\right)^{1 / 2}}\left(\frac{\partial \chi_{t-1}}{\partial q^{t-1}}\right)\right),
\end{aligned}
$$

where the derivatives need to be evaluated considering the unitary constraint on the quaternions parameters. With some simple algebra we finally obtain expressions 
for the torque on the bead $t$, about its $x, y$ and $z$ axes due to bead $t+1$, specifically

$$
\begin{aligned}
& t_{x}=\frac{2 I P}{\hbar^{2} \beta^{2}}\left(\frac{\Gamma(t+1, t)}{\left(1-\chi_{t+1}^{2}\right)^{1 / 2}}\left(-q_{3}^{t} q_{2}^{t+1}+q_{0}^{t} q_{1}^{t+1}+q_{2}^{t} q_{3}^{t+1}-q_{1}^{t} q_{0}^{t+1}\right)\right), \\
& t_{y}=\frac{2 I P}{\hbar^{2} \beta^{2}}\left(\frac{\Gamma(t+1, t)}{\left(1-\chi_{t+1}^{2}\right)^{1 / 2}}\left(-q_{0}^{t} q_{2}^{t+1}-q_{3}^{t} q_{1}^{t+1}+q_{1}^{t} q_{3}^{t+1}+q_{2}^{t} q_{0}^{t+1}\right)\right), \\
& t_{z}=\frac{2 I P}{\hbar^{2} \beta^{2}}\left(\frac{\Gamma(t+1, t)}{\left(1-\chi_{t+1}^{2}\right)^{1 / 2}}\left(q_{1}^{t} q_{2}^{t+1}-q_{2}^{t} q_{1}^{t+1}+q_{0}^{t} q_{3}^{t+1}-q_{3}^{t} q_{0}^{t+1}\right)\right) .
\end{aligned}
$$

Obviously, analogous terms arise due to the bead $t-1$.

It is worth noting that the final form for the torques depends on the particular convention chosen for the description of the rotational motion. This dependence is taken into account through the matrix $\Xi$ in equation (9), all other quantities being otherwise independent of the convention (see Appendix A). If the rotation is not free, the torque acting on bead $t$ of molecule $i$ will also include a torque due to an external potential (in the condensed phase this will be the intermolecular potential that is calculated every time step in any standard MD program). We have observed that the quantum torques alone (as given by equation (11)) naturally conserve the centroid (as do the quantum forces for a translational centroid). Thus it is only contributions due to the external potential that should ideally cause displacements of the (orientational) centroid.

\subsection{The orientational centroid}

Utilizing the torques derived above, together with those due to the external potential, in conjunction with the centroid constraint, a dynamics is generated such that the 'orientational neighbourhood' is sampled and the average centroid torque estimated. In this way the Cartesian calculation of a standard CMD simulation can be precisely mimicked. It is clear, however, that within an MD simulation the computer does not perform true infinitesimal rotations. As a consequence a small error arises associated with the preservation of the orientational centroid and as the bead dynamics proceed the real centroid drifts slightly from the original or ideal centroid. This error can be eliminated by exploiting the geometric properties of the quaternion parameters together with the aid of a definition for the orientational centroid. The question of how to conserve an orientational centroid is then posed, without loss of generality, in the following form: if we let the centroid be the quaternion $(1,0,0,0)$, can we find a set of bead orientations that satisfies this centroid?
This problem is quickly solved by representing the orientation of one bead in terms of the orientations of the remaining beads. The two-bead case is straightforward; the beads are inverse quaternions of each other, $q_{2}=q_{1}^{-1}$ (this case becomes obvious because it reduces to a one-dimensional problem, involving a single rotation angle). A simple proof can be given as follows: let us define a transition quaternion, $q_{t}$, that transforms $q_{1}$ into $q_{2}$, as $q_{2}=q_{t} q_{1}$. Then, since $q_{2}=q_{1}^{-1}$, we have $q_{t}=q_{2}\left(q_{1}\right)^{-1}=q_{2} q_{2}$, which means that in order to transform $q_{1}$ into $q_{2}$, two identical transformations need to occur. It should be intuitively clear that the centroid defined by two orientations is some orientation located symmetrically between them. Thus, we find that $q_{\mathrm{c}}=\sqrt{q_{t}} q_{1}=q_{2} q_{1}=\overline{1}$, where $\overline{1}$ is the unit quaternion $(1,0,0,0)$. The problem of determining the centroid defined by two (and in general $P$ ) arbitrary orientations is addressed later in this paper, but at this point it should be clear geometrically that if those two beads are inverse quaternions of each other their centroid is the quaternion $(1,0,0,0)$.

A solution for the three-bead case begins by defining

$$
q_{1}=\cos \left(\theta_{1} / 2\right)+\mathbf{u}_{1} \sin \left(\theta_{1} / 2\right)
$$

and

$$
q_{2}=\cos \left(\theta_{2} / 2\right)+\mathbf{u}_{2} \sin \left(\theta_{2} / 2\right),
$$

where $\theta_{1}$ and $\theta_{2}$ are the rotation angles and $\mathbf{u}_{1}$ and $\mathbf{u}_{2}$ are the rotation axes of the quaternions $q_{1}$ and $q_{2}$, respectively. It is interesting to examine the relationships for two simple cases.

(1) If $\mathbf{u}_{1}=\mathbf{u}_{2}=\mathbf{u}$, then the rotation axis of the third bead $\mathbf{u}_{3}=-\mathbf{u}$ and its rotational angle $\theta_{3}=\theta_{1}+\theta_{2}$. Furthermore, if additionally $\theta_{1}=-\theta_{2}$, the quaternion $q_{3}$ becomes the unit quaternion, i.e. the centroid itself.

(2) If the angle between $\mathbf{u}_{1}$ and $\mathbf{u}_{2}$ is $2 \pi / 3$ and $\theta_{1}=\theta_{2}=\theta$, then $\theta_{3}=\theta$ and $\mathbf{u}_{3}=-\mathbf{u}_{1}-\mathbf{u}_{2}$.

In general, it can be shown that the third quaternion (that satisfies the centroid) is given by

$$
q_{3}=\cos \left(\theta_{3} / 2\right)+\mathbf{u}_{3} \sin \left(\theta_{3} / 2\right),
$$

where

$$
\theta_{3}=\left[\theta_{1}^{2}+\theta_{2}^{2}+2 \theta_{1} \theta_{2}\left(\mathbf{u}_{1} \cdot \mathbf{u}_{2}\right)\right]^{1 / 2}
$$

and

$$
u_{3}=-\frac{\left[\theta_{1} / \sin \left(\theta_{1} / 2\right)\right] \mathbf{u}_{1}+\left[\theta_{2} / \sin \left(\theta_{2} / 2\right)\right] \mathbf{u}_{2}}{\left|\left[\theta_{1} / \sin \left(\theta_{1} / 2\right)\right] \mathbf{u}_{1}+\left[\theta_{2} / \sin \left(\theta_{2} / 2\right)\right] \mathbf{u}_{2}\right|} .
$$


We note that due to the cyclic nature of the rotations, equations (12) can become ill-conditioned if all the orientations are not confined to one hemisphere [12]; this limitation however happens not to be a problem in simulations closed to the classical limit where the quantum rotational uncertainty is small.

In principle, relations similar to equations (12) could be found for more than three beads; however, iterative procedures based on the two-bead and three-bead expressions are easily implemented for an arbitrary (but still relatively small) number of beads. For example, let $\left\{q_{t}\right\}$ be a set of $P$ orientations. From $q_{1}$ and $q_{2}$ and using the three-bead formula (equations (12)) we find a third (temporary) quaternion, denoted here by $q_{\text {temp. }}$. It is then clear for the case $P=3$ that $q_{3}=q_{\text {temp. }}$. From the discussion of the two-bead case this result implies that the influence on the centroid of the quaternions $q_{1}$ and $q_{2}$ is equivalent to (or can be substituted by) a single bead, specifically $\left(q_{\text {temp }}\right)^{-1}$, which obviously, together with $q_{3}=q_{\text {temp }}$ conserves the centroid $(1,0,0,0)$. For the case of four beads, we proceed in the same fashion. First we calculate $q_{\text {temp }}$ from the beads $q_{1}$ and $q_{2}$, then using $\left(q_{\mathrm{temp}}\right)^{-1}$ and $q_{3}$ (and equations (12)) we calculate a new $q_{\text {temp }}$ which in this case equals $q_{4}$. In general, for $P$ beads it is necessary to calculate $q_{\text {temp }} P-2$ times. The first $q_{\text {temp }}$ is calculated from $q_{1}$ and $q_{2}$ as before, and subsequent $q_{\text {temp }}$ are calculated by using $\left(q_{\text {temp }}\right)^{-1}$ from the previous step and the next bead, finally at the last step $q_{P}=q_{\text {temp. }}$. This procedure can then be used in addition to the dynamical constraint on the torques described above (see equation (7a)) to guarantee conservation of the centroid during the integration of the beads' equations of motion. We also remark that the above procedure could be utilized in a Monte Carlo algorithm to determine sets of bead quaternions that satisfy the centroid thereby providing an alternate means for the evaluation of the constrained path integral.

It is now interesting to return to answer the more general question, what is the orientational centroid for a given set of $P$ orientations, $\left\{q_{t}\right\}$ ? After careful consideration, it becomes apparent that the orientational centroid can be defined as that orientation, $q_{\mathrm{c}}$, that satisfies

$$
\min \left[\sum_{t=1}^{P} \Gamma^{2}\left(q_{\mathrm{c}}, q_{t}\right)\right]
$$

where $\Gamma\left(q_{\mathrm{c}}, q_{t}\right)$ is the rotation angle (or arc length) between the centroid orientation and the orientation of bead $t, q_{t}$. It is interesting to point out that a generalized form of this result has been recently proven [12]. With this definition one can obviously find the centroid of a set of orientations through a Monte Carlo search that uses this minimization condition as a criterion for rejection/ acceptance of trial centroid moves. Convergence is then achieved by finding that trial centroid that can no longer be improved. Such a search procedure can allow one to check the centroid conservation at any stage of a CMD simulation; in this study it was used to confirm the validity of the quaternion formulas given in equations (12). A similar Monte Carlo search procedure could also be used in a general context to find an average orientation or centroid. In the most general case, however, the minimization algorithm may require specific modifications that help it to avoid possible local minima and to handle possible degenerate solutions.

\section{Results and discussion}

We turn now to explicit results of calculations designed to demonstrate the success of the methodology described in the previous section. An isolated TIP4P [13] water molecule coupled to a thermal bath at $298 \mathrm{~K}$ is examined in the absence and presence of an external homogeneous electric field. The electric field is defined to be in the $z$ direction and is approximately equal in magnitude to the average local field present in liquid water $\left(\sim 2 \mathrm{~V}^{-1}\right)[14]$. The required path integral evaluation was carried out for different numbers of beads (between three and six) and in every case each bead was coupled to a Nosé-Hoover chain thermostat [15] of length 4. The simulation utilizes 256 otherwise independent water molecules being controlled with a single thermostat [16] to allow for appropriate fluctuations in the single-molecule kinetic energies in field-on conditions. The equations of motion were integrated using a fourth-order Gear predictor-corrector algorithm [16] and a real time step size of 1 and $0.125 \mathrm{fs}$ for the classical and the quantum simulations, respectively. Further computational details are given elsewhere [17].

Figure 1 shows the time behaviour of the instantaneous rotation angle (averaged over the five beads of all 256 molecules) between the beads and the centroid orientation for a field-free isolated molecule. The drift in the centroid orientation (manifest as a consistently increasing value for the bead-centroid arc length) clearly observed in figure $1(a)$, where only the conditions required by equations (7) and equation (11) have been utilized, is due to the non-commutativity of finite rotations. In figure $1(b)$ this drift has been eliminated by the further application (to each set of five beads) of the iterative procedure discussed in the context of equations (12). In this study it has been implemented by correcting a randomly selected bead orientation at every step of the constrained dynamics. It should be noted that the correction required for each step is always small (as implied by figure $1(a)$ ); consequently its impact 

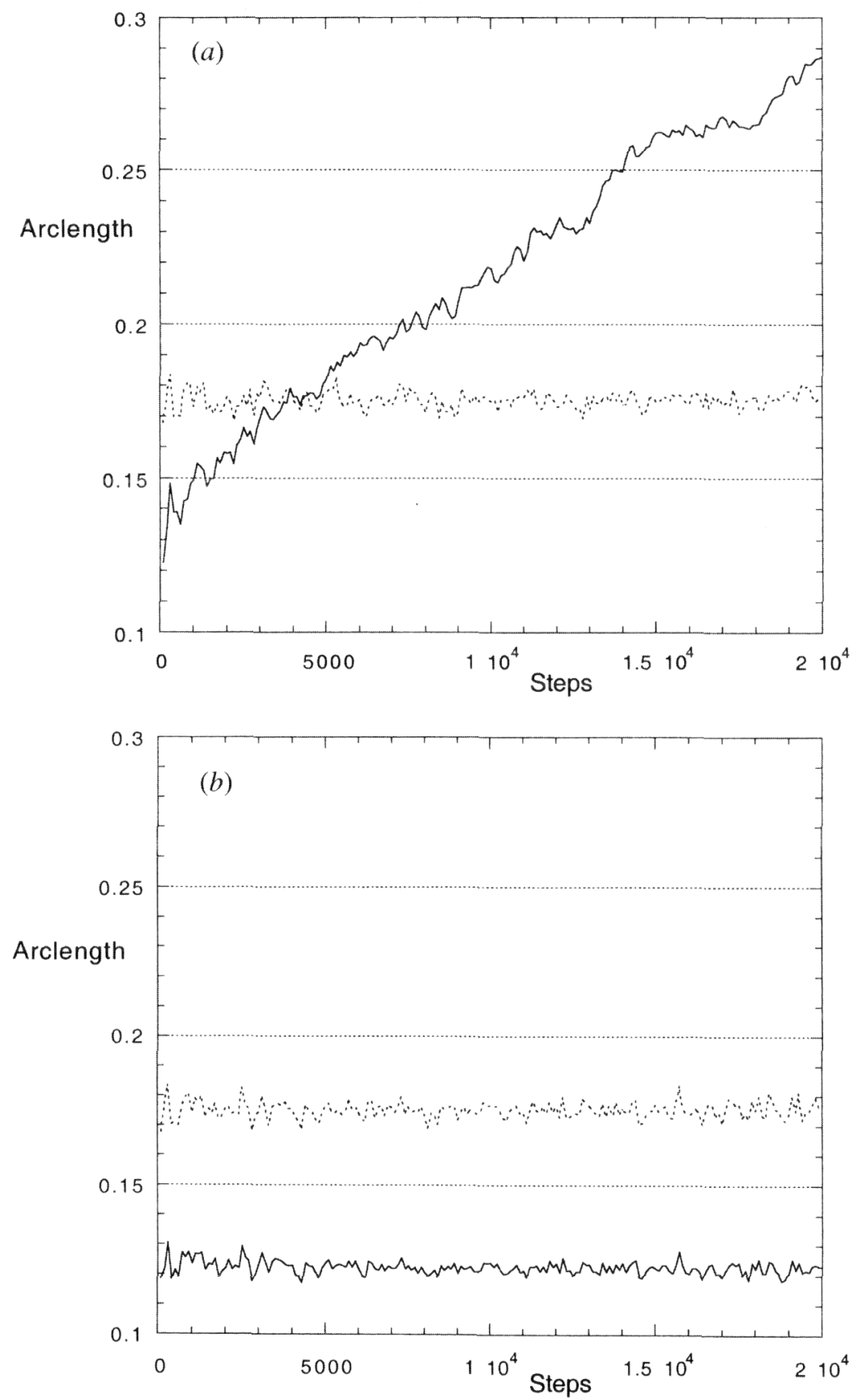

Figure 1. Time dependence of the average rotation angle between the beads, and between the beads and the centroid. The solid lines correspond to the bead-centroid angle and the dashed lines correspond to the bead-bead angle. (a) Results from dynamics utilizing only equations (7) and (11). (b) Results obtained utilizing equations (7), (11) and (12).

on the equations of motion is small and any heating effects are easily controlled by the thermostat. We have chosen to correct for the centroid at every time step to minimize such effects.

A requirement for CMD is a homogeneous sampling of the imaginary time phase space. Figure 2 shows probability distributions representing the quantum mechanical rotational uncertainty of a water molecule as mapped by the algorithm in the absence and presence of the electric field. The discrete values from the simulation appear to follow exactly the continuous lines that represent the expected normalized Gaussian form (including its angular differential element). In figure 2 we also see that the influence of the field is to 


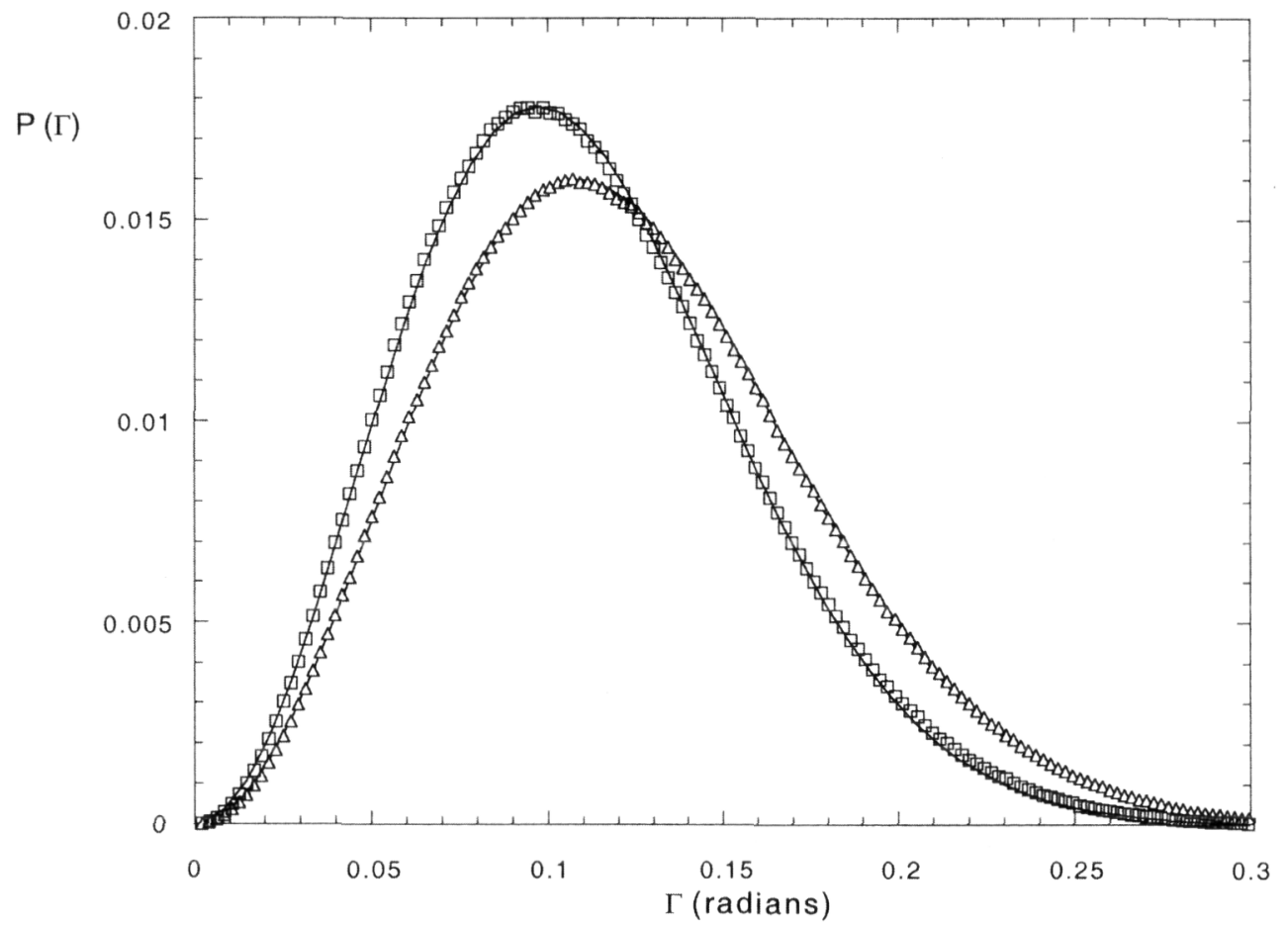

Figure 2. Probability distribution of the bead-centroid angle of a water molecule. The triangles represent results from a simulation carried out in the absence of field and the squares are results obtained in the presence of field. The lines are the ideal distributions fit to the data.

slightly contract the quantum mechanical angular distribution (i.e. as expected the external potential slightly contracts the quantum uncertainty).

The probability distribution for the orientation of the molecular dipole moment in the presence of the external field and its dependence on $P$, the discretization parameter, is shown in figure 3, where $\alpha$ is the angle between the dipole moment and the applied field. As can be seen in figure $3(a)$, results from quantum calculations with different values of $P$ demonstrate that convergence is achieved at rather low values of the discretization parameter, in particular, the results at $P=5$ become indistinguishable from those at $P=6$. Figure $3(b)$ compares the probability distributions for the orientation of the dipole moment obtained from classical and quantum simulations. The broadening observed in the distribution from the quantum system is clearly a manifestation of quantum dispersion.

One of the principal benefits of the CMD approach is in its ability to characterize the quantum dynamics. The application of CMD to translational degrees of freedom has established a strategy for the calculation of (approximate) quantum time correlation functions [5-7]. This strategy (see equation (3)) can be applied in the context of the rotational centroid provided that the system is close to the classical limit and the interactions are not strongly anharmonic [7]. The classical and quantum time correlation functions for the $x$ component of the angular velocity for water molecules in field-on conditions are shown in figure 4. As expected we see that the effect is to reduce the structure in this function.

In order to consider a more realistic application for the present methodology, a preliminary liquid water simulation at $298 \mathrm{~K}$ has been performed. The TIP4P water model with a discretization parameter, $P$, of 5 was used. (A complete description of these calculations and their results will be given in a forthcoming article [17].) Figure 5 illustrates the convergence of the forces and torques within a CMD simulation where the axes and the molecules have been selected at random. The relative errors shown in figure 5 are the relative differences between the current running average and the 'exact' (long-time) average, where the 'exact' values were determined from a simulation consisting of $10^{5}$ imaginary steps. We see that to obtain reasonably converged forces and torques a few thousands steps are required in the imaginary time sampling. This makes the application of the so-called 'primitive' CMD approach (where well-averaged forces and torques are evaluated for each centroid step [18]) computationally very demanding; however, utilization of the 'adiabatic' CMD approach [17] requires considerably less computational effort to produce a smooth dynamical trajectory. It is also 

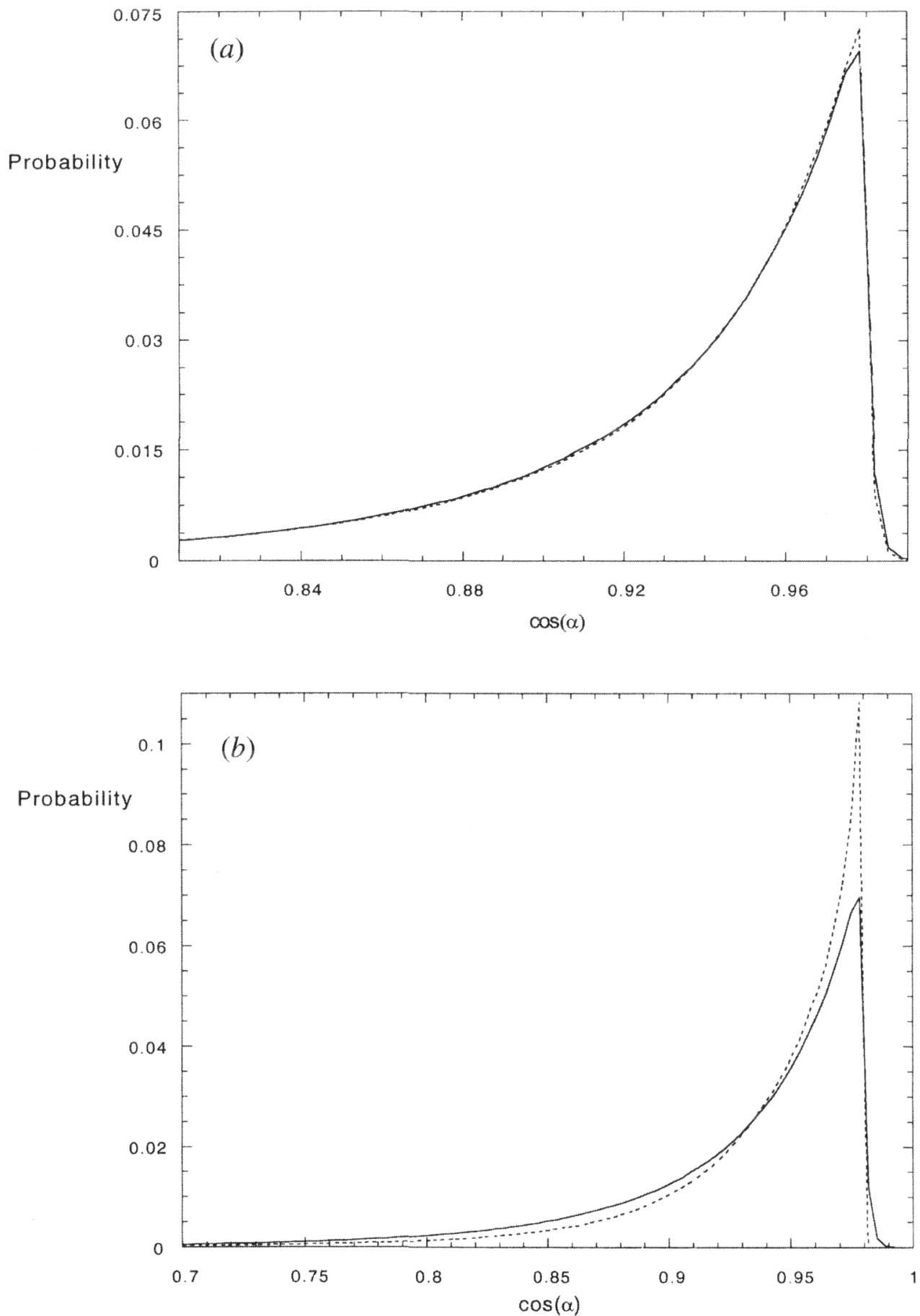

Figure 3. Dipole moment orientational distributions of the water molecule as a function of $\cos (\alpha)$, where $\alpha$ is the angle between the dipole and the field vector. (a) Results for quantum mechanical calculations at different $P$. The solid, dot-dashed and dashed lines represent $P=6, P=5$ (which overlaps with $P=6$ line) and $P=3$, respectively. (b) Classical and quantum distributions. The solid line is the quantum result while the dashed line is from the classical simulation.

evident from figure 5 that the centroid torques converge more rapidly than the centroid forces.

In summary, we have extended the CMD method to rotations of rigid bodies by designing an algorithm that in the context of a molecular dynamics simulation samples homogeneously the orientational neighbourhood associated with their quantum degrees of freedom. This algorithm ensures that the rotational centroid (or the 
The rotational centroid and its application in quantum molecular dynamics simulations

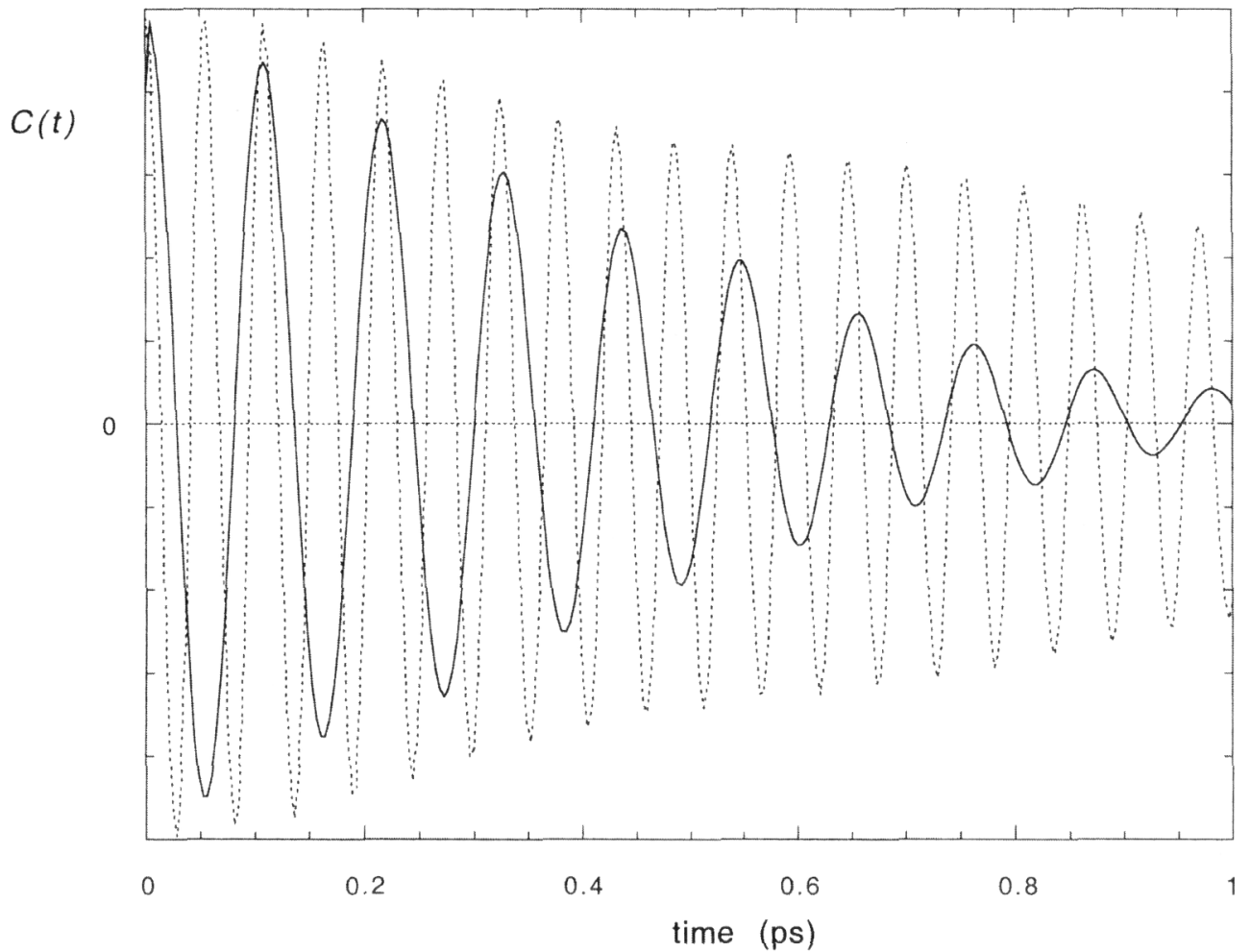

Figure 4. Classical (dashed line) and quantum (solid line) normalized time correlation functions for the $x$ component of the angular velocity.

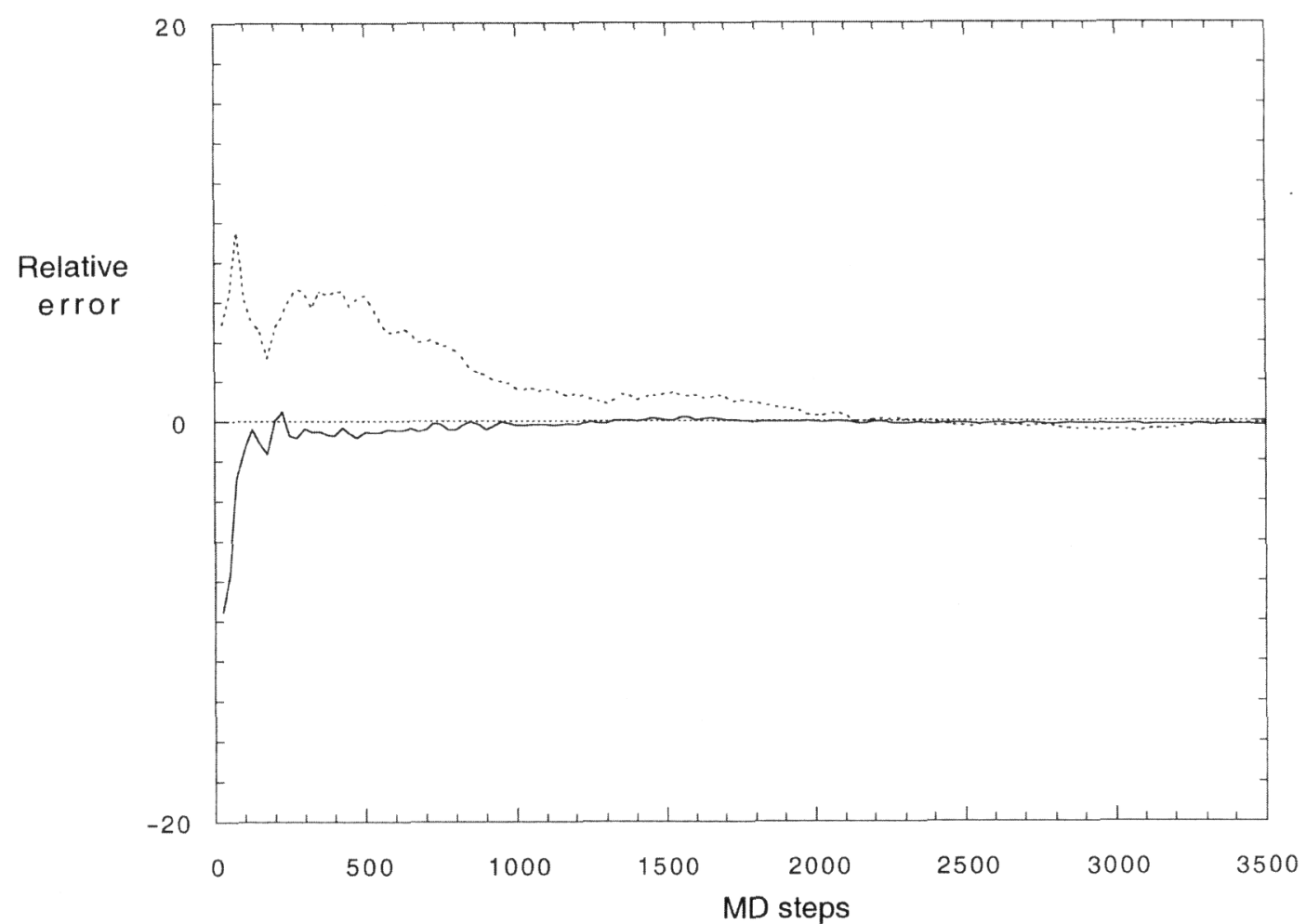

Figure 5. Convergence of the centroid force and the centroid torque on a molecule in liquid water as a function of the MD imaginary time sampling. The dashed line represents a randomly selected component of the force and the solid line represents a randomly selected component of the torque. 
average orientation of the set of beads) remains constant at each step. To help achieve this, we have provided a general definition for the average orientation (rotational centroid) and a straightforward procedure for determining it numerically. The methodology we have presented here will be particularly advantageous for the quantum simulation of the dynamical properties of (simple) molecular systems in condensed phases and near their classical limit (such as liquid water) within the rigid body approximation as will be demonstrated in a forthcoming article [17].

We are grateful for the financial support of the Natural Sciences and Engineering Research Council of Canada.

\section{Appendix A}

This appendix presents a simple and straightforward derivation of the rotational equations of motion in quaternion form. The authors are aware that those equations are not new (see for example [11]), but find the development helpful to stress the simplicity of the quaternion approach to rotational motion and, in particular, the specification of the structure of the torques in quaternion form.

Let us consider the rotational motion of a rigid body. In this case, the Lagrangian becomes

$$
\mathcal{L}=K-V=\frac{1}{2} \sum_{i} I_{i} w_{i}^{2}-V,
$$

where $K$ is the rotational kinetic energy and $V$ is the interaction potential (which depends only on the orientation of the rigid body). If we choose the quaternion parameters as the generalized coordinates, Lagrange's equation for the coordinate $q_{j}$ is

$$
\frac{\partial \mathcal{L}}{\partial q_{j}}-\frac{\mathrm{d}}{\mathrm{d} t} \frac{\partial \mathcal{L}}{\partial \dot{q}_{j}}=0
$$

which can be expressed as

$$
\sum_{i=1}^{3} \frac{\partial K}{\partial w_{i}} \frac{\partial w_{i}}{\partial q_{j}}-\frac{\partial V}{\partial q_{j}}-\frac{\mathrm{d}}{\mathrm{d} t}\left(\sum_{i=1}^{3} \frac{\partial K}{\partial w_{i}} \frac{\partial w_{i}}{\partial \dot{q}_{j}}-\frac{\partial V}{\partial \dot{q}_{j}}\right)=0
$$

where $w_{i}$ are the angular velocities and the sum is over the components $x, y, z$. Equation (A 3) reduces to

$$
\sum_{i=1}^{3} \frac{\partial K}{\partial w_{i}} \frac{\partial w_{i}}{\partial q_{j}}-\frac{\mathrm{d}}{\mathrm{d} t}\left(\sum_{i=1}^{3} \frac{\partial K}{\partial w_{i}} \frac{\partial w_{i}}{\partial \dot{q}_{j}}\right)=\frac{\partial V}{\partial q_{j}}
$$

due to the fact that the potential energy does not depend on any 'velocity', $\partial V / \partial \dot{q}_{j}=0$, it depends only on the orientation.

It is well known that the principle angular velocities are related to the time derivatives of the quaternions [11]:

$$
\begin{gathered}
{\left[\begin{array}{c}
w_{1} \\
w_{2} \\
w_{3} \\
0
\end{array}\right]=2\left[\begin{array}{cccc}
-q_{3} & q_{0} & q_{2} & -q_{1} \\
-q_{0} & -q_{3} & q_{1} & q_{2} \\
q_{1} & -q_{2} & q_{0} & -q_{3} \\
q_{2} & q_{1} & q_{3} & q_{0}
\end{array}\right]\left[\begin{array}{c}
\dot{q}_{2} \\
\dot{q}_{1} \\
\dot{q}_{3} \\
\dot{q}_{0}
\end{array}\right]} \\
=2 \Xi \dot{q}=w .
\end{gathered}
$$

(Equation (A 5) can be obtained directly from the definition of the derivative of a quaternion and the appropriate quaternion convention, i.e. $q=q_{0}+$ $i q_{1}-j q_{2}+k q_{3}$.) If we differentiate the components of $w$ in (A 5) with respect to $q_{j}$ and $\dot{q}_{j}$, substitute in equation (A 4) and use $\partial K / \partial w_{i}=I_{i} w_{i}=L_{i}$ for $j=0,1,2,3$, we have

$$
\begin{aligned}
& 4\left[\begin{array}{cccc}
\dot{q}_{1} & -\dot{q}_{2} & \dot{q}_{3} & \pm \dot{q}_{0} \\
-\dot{q}_{0} & \dot{q}_{3} & \dot{q}_{2} & \pm \dot{q}_{1} \\
\dot{q}_{3} & \dot{q}_{0} & -\dot{q}_{1} & \pm \dot{q}_{2} \\
-\dot{q}_{2} & -\dot{q}_{1} & -\dot{q}_{0} & \pm \dot{q}_{3}
\end{array}\right]\left[\begin{array}{c}
L_{1} \\
L_{2} \\
L_{3} \\
0
\end{array}\right] \\
& +2\left[\begin{array}{cccc}
q_{1} & -q_{2} & q_{3} & \pm q_{0} \\
-q_{0} & q_{3} & q_{2} & \pm q_{1} \\
q_{3} & q_{0} & -q_{1} & \pm q_{2} \\
-q_{2} & -q_{1} & -q_{0} & \pm q_{3}
\end{array}\right]\left[\begin{array}{c}
\dot{L}_{1} \\
\dot{L}_{2} \\
\dot{L}_{3} \\
0
\end{array}\right]=\left[\begin{array}{c}
\partial V / \partial q_{0} \\
\partial V / \partial q_{1} \\
\partial V / \partial q_{2} \\
\partial V / \partial q_{3}
\end{array}\right],
\end{aligned}
$$

where $L_{i}$ is clearly a component of the angular momentum vector. From equation (A 6) it is easy to obtain the equations of motion, which can be expressed in quaternion form as

$$
\dot{L}=\frac{1}{2} \Xi \cdot N-2 \Xi \cdot \dot{\Xi}^{\mathrm{T}} \cdot L,
$$

where

$$
\dot{L}=\left[\begin{array}{c}
\dot{L}_{1} \\
\dot{L}_{2} \\
\dot{L}_{3} \\
0
\end{array}\right], \quad N=\left[\begin{array}{l}
-\partial V / \partial q_{2} \\
-\partial V / \partial q_{1} \\
-\partial V / \partial q_{3} \\
-\partial V / \partial q_{0}
\end{array}\right], \quad L=\left[\begin{array}{c}
L_{1} \\
L_{2} \\
L_{3} \\
0
\end{array}\right]
$$

Equation (A 7) was published and used by Evans and co-worker [11]. He observed that the evolution of the angular momentum can be determined knowing the initial angular momentum, the particular orientation of the rigid body and having evaluated the derivatives 
in $N$. It is clear then that the principal torques in quaternion form, $T$, are defined by

$$
T=\frac{1}{2} \Xi \cdot N \text {. }
$$

As previously noted by Evans and co-worker [11], the quaternion parameters, in contrast to Euler angles, give equations of motion without singularities. This is simply due to the fact that $\Xi$ only becomes singular when $q_{0}^{2}+q_{1}^{2}+q_{2}^{2}+q_{3}^{2}=0$, but this never occurs because of the quaternion unitary constraint $q_{0}^{2}+q_{1}^{2}+$ $q_{2}^{2}+q_{3}^{2}=1$.

\section{References}

[1] Feynman, R. P., and Hibis, A. R., 1965, Quantum Mechanics and Path Integrals (New York: McGraw-Hill); Feynman, R. P., 1972, Statistical Mechanics: a Set of Lectures (Redwood City, CA: Addison-Wesley).

[2] Berne, B. J., and Thirumalai, D., 1986, Annu. Rev. phys. Chem., 37, 401; Chandler, D., 1991, Liquids, Freezing and Glass Transition, edited by J. P. Hansen, D. Levesque and J. Zinn-Justin (Amsterdam: North-Holland).

[3] Parinello, M., and Rahman, A., 1984, J. chem. Phys., 80, 860; Walqvist, A., and Berne, B. J., 1985, Chem. Phys. Lett. 117, 214

[4] Kuharski, R. A., and Rossky, P. J., 1985, J. chem. Phys., 82, 3728; Del Buono, G. S., Rossky, P. J., and SCHNitKER, J., 1991, ibid., 95, 3728.

[5] Lobaough, J., and Voth, G. A., 1997, J. chem. Phys., 106, 2400.

[6] Voth, G., 1996, Advances in Chemical Physics, Vol. XCIII, edited by I. Prigogine and S. A. Rice (New York: John Wiley \& Sons); Voth, G. A., 2000, Theoretical Methods in Condensed Phase Chemistry, edited by S. D.
Schwartz (Dordrecht, Boston: Kluwer Academic Publishers)

[7] Cao, J., and Voth, G. A., 1993, J. chem. Phys., 99, 10070; 1994, ibid., 100, 5093; 1994, ibid., 100, 5106; 1994, ibid., 101, 6157; 1994, ibid., 101, 6168; JANG, S., and VoTH, G. A., 1999, ibid., 111, 2357; 1999, ibid., 111, 2371.

[8] Wallovist, A., and Mountain, R. D., 1999, Reviews in Computational Chemistry, Vol. 13, edited by K. B. Lipkowitz and D. B. Boyd (New York: John Wiley \& Sons); Guillot, B., 2002, J. molec. Liq., 101, 219; Kusalik, P. G., and Svishchev, I. M., 1994, Science, 265, 1219

[9] For a review on the use of path integrals in rigid rotors see Marx, D., and Müser, M. H., 1999, J. Phys.: condens. Matter, 11, R117.

[10] Schulman, L. S., 1981, Techniques and Applications of Path Integration (New York: John Wiley \& Sons).

[11] Evans, D. J., and Murad, S., 1989, Molec. Phys., 68, 6; Evans, D. J., 1977, ibid., 34, 317; Evans, D. J., and Murad, S., 1977, ibid., 34, 327.

[12] Buss, S. R., and Fillmore, J. P., 2001, ACM T Graphic, 20, 95.

[13] Jogensen, W. L., Chandresekhar, J., Madura, J. D., ImPley, R. W., and Klein, M. L., 1983, J. chem. Phys., 79, 926.

[14] Gubskaya, A. V., and Kusalik, P. G., 2002, J. chem. Phys., 117, 5290

[15] Martina, G. J., and Klein, M. L., 1992, J. chem. Phys., 97, 2635.

[16] Allen, M. P., and Tildesley, D. J., 1989, Computer Simulation of Liquids (New York: Oxford University Press).

[17] Hernandez de la Pẽna, L., and Kusalik, P. G., 2004, J. chem. Phys., submitted.

[18] Pavese, M., Jang, S., and Voth, G. A., 2000, Pararell Comput., 26, 1025. 